\title{
PRAXIS
}

sanjs

\section{PROCESO DE ENFERMERÍA APLICADO A USUARIO POST-OPERADO DE LAPAROTOMÍA EXPLORATORIA}

\author{
NURSING PROCESS APPLIED TO POST-OPERATED USER OF EXPLORATORY LAPAROTOMY
}

ROJAS VEGA MARÍA EMMA ${ }^{1 *}$, SANDOVAL GUTIÉRREZ KARLA ABIGAIL ${ }^{2}$

\begin{abstract}
RESUMEN:
ntroducción: el presente estudio de caso expone a un usuario post-operado de laparotomía exploratoria, hospitalizado en el servicio de Unidad de Cuidados Intensivos en la ciudad de HermosiIlo, Sonora, México. Objetivo: exhibir la estructura que permitió cubrir las necesidades individuales del usuario en cuestión. Metodología: se realizó una valoración de Enfermería focalizada de acuerdo a la Guía de Valoración de Enfermería según la tipología de patrones funcionales de M. Gordon para persona adulta. Así mismo, se empleó la taxonomía NANDA-I: Definiciones, taxonomía NOC para la Clasificación de Resultados de Enfermería y taxonomía NIC para la Clasificación de Intervenciones de Enfermería para la planeación de intervenciones y actividades. También se recuperó información del expediente clínico de la unidad hospitalaria. Resultados: se identificaron un total de 10 diagnósticos de enfermería, de los cuales cuatro son diagnósticos reales y los restantes de riesgo. Conclusiones: es vital que el personal de enfermería implemente todos sus conocimientos y habilidades en la aplicación de sus intervenciones durante cada una de las etapas del PE, de esa manera se logrará brindar un cuidado enfermero de calidad y completamente fundamentado.
\end{abstract}

Palabras clave: Proceso de enfermería, estudio de caso, unidad de cuidados intensivos, laparotomía (DeCS, BIREME).

\section{ABSTRACT}

Introduction: the present case study exposes a post-operative exploratory laparotomy user, hospitalized at the Intensive Care Unit senvice in the city of Hermosillo, Sonora, Mexico. Objective: exhibit the structure that allowed covering the individual needs of the user in question. Methodology: a focused nursing assessment was carried out according to M. Gordon's Nursing Assessment Guide of functional patterns typology for an adult person. Likewise, Nursing Diagnoses were used: Definitions and Classification for the selection of nursing diagnoses, as well as the Nursing Results Classification and Nursing Interventions Classification for the planning of interventions and activities. Information was also retrieved from the clinical file of the hospital unit Results: a total of 10 nursing diagnoses were identified, of which four are real diagnoses and the rest are risk diagnoses. Condusions: it is vital that the nursing staff implements all their knowledge and skills in the application of their interventions during each stage of the NP, by this way it will be possible to provide a quality and completely fundamental nursing care.

Key words: Nursing process, case study, intensive care unit, laparotomy (DeCS, BIREME).

\footnotetext{
${ }_{1 *}^{1 *}$ asante de Licenciatura en Enfermería. Departamento de Enfermería. Universidad de Sonora. E-mail: mariaemmarove@hotmail.com

${ }^{1}$ Pasante de Licenciatura en Enfermería. Departamento de Enfermería. Universidad de Sonora.
} 


\section{INTRODUCCIÓN}

El Proceso de Enfermería (PE) es un método sistemático y racional de planificar y brindar cuidados, cuyo objetivo es identificar las necesidades de salud actuales y potenciales para establecer planes sobre las necesidades identificadas y actuaciones específicas. Es un todo cíclico, dinámico e inseparable, estructurado en secuencias lógicas. Para la correcta aplicación del PE se ha de considerar el modelo teórico o conceptual que se haya elegido como marco de referencia, este fundamentará la intervención de Enfermería en cada una de las fases del proceso(1). Por otra parte, un estudio de caso es un método de aprendizaje acerca de una situación compleja; se basa en el entendimiento, el cual se obtiene a través de la descripción y análisis en su conjunto y dentro de su contexto(2).

Adentrándonos al contexto en el cual se ha llevado acabo el presente trabajo, podemos definir a la Unidad de Cuidados Intensivos como un área de hospitalización, en la que un equipo multi e interdisciplinario brinda atención médica a usuarios en estado crítico, quienes presentan alteraciones de los principales sistemas fisiológicos, los cuales requieren asistencia continúa y son recuperables ${ }^{(3)}$. El presente estudio de caso tiene la finalidad de exponer un PE focalizado, aplicado a usuario post-operado de laparotomía exploratoria en $\mathrm{UCl}$.

\section{METODOLOGÍA}

Se utilizó la Guía de Valoración de Enfermería según tipología de patrones funciones de salud de M. Gordon para persona adulta para la realización de la primera etapa del PE. Posteriormente, se empleó Diagnósticos Enfermeros: Definiciones y Clasificación ${ }^{(4)}$ para la selección de diagnósticos, así como la Clasificación de Resultados de Enfermería ${ }^{(5)}$ y Clasificación de Intervenciones de Enfermería ${ }^{(6)}$ para la realización de las últimas etapas. Así mismo, se recuperó información del expediente clínico de la unidad hospitalaria debido al estado de salud del usuario, ausencia de familiares y a los horarios de la práctica clínica.

\section{PRESENTACIÓN DEL CASO}

\section{Datos sociales básicos}

Hombre de 67 años de edad, ingresa el día 23 de agosto del 2016 al servicio de Urgencias por motivo de dolor abdominal generalizado y ausencia de evacuación intestinal, iniciando signos y síntomas el mismo día; reside en la ciudad de Hermosillo, Sonora, México. Actualmente se encuentra post-operado de laparotomía exploratoria en $\mathrm{UCl}$, ingresando el día 30 de Agosto del 2016. Se realiza valoración del estado de salud a partir del día 31 de agosto del 2016 a las siete de la mañana dentro de la unidad hospitalaria, culminando el día 02 de septiembre del 2016 a las 12 horas.

\section{Antecedentes personales de relevancia}

Hipertensión Arterial (HTA) desde hace 12 años sin tratamiento; Diabetes Mellitus II (DM II) hace 18 años con tratamiento de Insulina Glargina 40 unidades y Metformina 850 mg; Cáncer de Recto (CR) sin más datos al respecto y presenta toxicomanías. Transfusiones positivas el día 30 de agosto del 2016. En cuanto a tratamientos quirúrgicos, se hace mención a laparotomía exploratoria realizada el día 30 de agosto del 2016.

\section{Valoración focalizada de enfermería por patrones funcionales de M. Gordon}

\section{Resumen de inicio y evolución del padecimiento actual}

El día 23 de agosto del 2016 inicia con dolor abdominal y ausencia de evacuaciones, por lo que acude al servicio de Urgencias, en el cual es diagnosticado con obstrucción intestinal y referido al servicio de Medicina Interna (MI). El día 30 de agosto del 2016 se realiza laparotomía exploratoria encontrándose líquido cetrino, por lo cual, se efectúa un aseo de cavidad abdominal, al igual que un desmantelamiento de anastomosis y colostomía; al término de la cirugía se traslada al servicio de UCl. Al día de 31 de agosto del 2016, según el score Acute Physiology and Chronic Health Evaluation II, presenta una puntuación de 24, lo cual se clasifica en APACHE grado V con un pronóstico de mortalidad de $56.94 \%$.

\section{Tratamientos farmacológicos}

\begin{tabular}{|c|c|c|c|}
\hline \multicolumn{4}{|c|}{ Patrón Nutricional Metabólico } \\
\hline Medicamento & Dosis & Vía & Frecuencia \\
\hline Albumina & 1 frasco & IV & $166 \mathrm{ml} / \mathrm{hr}$ \\
\hline $\begin{array}{l}\text { Gluconato de } \\
\text { Calcio }\end{array}$ & $\begin{array}{l}1 \text { ám- } \\
\text { pula }\end{array}$ & IV & $\begin{array}{c}\text { Cada } 8 \text { ho } \\
\text { ras }\end{array}$ \\
\hline Meropenem & $\begin{array}{l}1 \text { gra- } \\
\text { mo }\end{array}$ & IV & $\begin{array}{c}\text { Cada } 8 \text { ho } \\
\text { ras }\end{array}$ \\
\hline Metroclopramida & $\begin{array}{l}1 \text { ám- } \\
\text { pula }\end{array}$ & IV & $\begin{array}{c}\text { Cada } 8 \text { ho } \\
\text { ras }\end{array}$ \\
\hline Metronidazol & $750 \mathrm{mg}$ & IV & $\begin{array}{c}\text { Cada } 8 \text { ho } \\
\text { ras }\end{array}$ \\
\hline Omeprazol & $40 \mathrm{mg}$ & IV & $\begin{array}{c}\text { Cada } 24 \\
\text { horas }\end{array}$ \\
\hline Paracetamol & $\begin{array}{c}1 \text { gra- } \\
\text { mo }\end{array}$ & IV & PRN \\
\hline PFC & $\begin{array}{l}1 \text { uni- } \\
\text { dad }\end{array}$ & IV & $\begin{array}{c}\text { Cada } 8 \text { ho- } \\
\text { ras }\end{array}$ \\
\hline
\end{tabular}




\section{Esquema de Insulina (IAR)}

\begin{tabular}{ccccc}
\hline $\begin{array}{c}\text { Menor a } 180 \mathrm{mg} / \mathrm{dL}: 0 \\
\text { unidades }\end{array}$ & $\begin{array}{c}\text { De } 181 \mathrm{mg} / \mathrm{dL} \text { a } 250 \\
\mathrm{mg} / \mathrm{dl}: 4 \text { unidades }\end{array}$ & $\begin{array}{c}\text { De } 251 \mathrm{mg} / \mathrm{dL} \text { a } 300 \\
\mathrm{mg} / \mathrm{dL}: 6 \text { unidades }\end{array}$ & $\begin{array}{c}\text { De } 301 \mathrm{mg} / \mathrm{dL} \text { a } 350 \\
\mathrm{mg} / \mathrm{dL}: 8 \text { unidades }\end{array}$ & $\begin{array}{c}\text { Mayor a } 351 \mathrm{mg} / \mathrm{dL}: \\
10 \text { unidades }\end{array}$
\end{tabular}

\begin{tabular}{c|c|c|c}
\hline \multicolumn{5}{c}{ Patrón Eliminación } \\
\cline { 1 - 2 } Medicamento & Dosis & Vía & Frecuencia \\
\hline Furosemide & $20 \mathrm{mg}$ & IV & Cada 24 horas \\
\hline
\end{tabular}

\begin{tabular}{c|c|ccc}
\hline \multicolumn{2}{c}{ Patrón Actividad y Ejercicio } & & \\
Medicamento & Dosis & Vía & Frecuencia \\
\hline Enoxaparina & $80 \mathrm{mg}$ & SC & Cada 24 horas \\
Norepinefrina & $116 \mathrm{mg}$ & IV & $1.5 \mathrm{mcg} / \mathrm{kg} / \mathrm{min}$ \\
Vitamina K & 1 ámpula & IV & Cada 24 horas \\
\hline
\end{tabular}

\begin{tabular}{c|c|ccc}
\hline & \multicolumn{2}{c}{ Patrón Cognitivo Perceptivo } & & Velocidad \\
\hline Medicamento & Dosis & Vía & \\
Fentanilo & 4 ámpulas & IV & $\mathrm{mcg} / \mathrm{min}$ \\
Midazolam & $100 \mathrm{mg}$ & $\mathrm{IV}$ & & $20 \mathrm{mg} / \mathrm{min}$ \\
\hline
\end{tabular}

\section{Descripción de patrones de salud}

\section{Patrón Nutricional-Metabólico}

En ayuno desde hace tres días con un total de líquidos ingresados de $1222.5 \mathrm{ml} / 5 \mathrm{hr}$; Índice de Masa Corporal (IMC) indica Obesidad Tipo III $\left(41.01 \mathrm{~kg} / \mathrm{m}^{2}\right)$ según la NOM-043-SSA2-2012 (7). Normotérmico $\left(37^{\circ} \mathrm{C}\right)$, tegumentos y membranas mucosas pálidas con ligera ictericia. Edema con fóvea de una cruz en ambos pies, así como temperatura disminuida al tacto a diferencia del resto del cuerpo; uñas de extremidades inferiores con onicomicosis. Mucosa bucal pálida, dentadura con presencia de sarro y decoloración de esmalte con lengua saburral y saliva de consistencia espesa; presencia de tubo endotraqueal en buenas condiciones.

Abdomen globoso, distendido, asimétrico con ausencia de peristaltismo y con temperatura elevada al tacto; hipertensión intraabadominal grado II (Presión Intraabdominal (PIA) $18 \mathrm{mmHg}$ según expediente clínico) y deficiente presión de perfusión abdominal $(55 \mathrm{mmHg})$, lo cual sugiere un síndrome compartimental abdominal. Herida quirúrgica de 24 horas de evolución y en fase inflamatoria en zona media abdominal con una longitud de 20 centímetros; bordes no afrontados en su totalidad, presencia de escasa secreción sanguinolenta, rubor, tumefacción, temperatura elevada y con presencia de grapas. Mantiene sistema de drenaje tipo Blake en cuadrante superior derecho y de colostomía en cuadrante superior izquierdo ambas sin signos de infección y funcionales; esta última se encuentra edematizada y de coloración rosa brillante.

De acuerdo a exámenes de laboratorio de biometría hemática presenta en serie roja microcitosis, debido a una probable deficiencia de moléculas de oxígeno, lo cual se relaciona con la palidez de tegumentos y la temperatura disminuida en extremidades. En lo referente a la serie blanca presenta neutrofilia, lo cual guarda relación con el proceso de cicatrización de herida quirúrgica $(\mathrm{Hx} \mathrm{Qx})$; presenta linfocitopenia y esosinopenia, relacionado con CR.

En cuanto a química sanguínea, presenta hiperglucemia, relacionado con su patología de DM II y al estrés metabólico como respuesta fisiológica del organismo a la agresión. Según los laboratorios de perfil hepático presenta hiperbilurrubinemia, relacionado con la deficiencia de oxígeno y con la ictérica de tegumentos. Así mismo presenta proteínas totales bajas, lo cual guarda relación con la inmunodeficiencia que padece por CR. Procalcitonina elevada, clasificada en riesgo moderado de padecer infecciones bacterianas (Tabla 1).

Riesgo de úlceras por presión en alto riesgo según la escala de Norton (8 puntos).

\section{Patrón Eliminación}

Eliminación vesical con apoyo de sonda Foley e intestinal con apoyo de colostomía; micciones con presencia de 
hematuria en grado I (Hemostick) y ausencia de defecación desde su ingreso. Presencia de drenaje de líquido biliar por sonda nasogástrica a derivación, de característica amarilla oscura y drenaje Blake con secreción sanguinolenta. Diuresis horaria $(1.1 \mathrm{ml} / \mathrm{kg} / \mathrm{hr}$ ) y diuresis minuto (2 $\mathrm{ml} / \mathrm{min}$ ) dentro de los parámetros normales (Pérdidas insensibles de $262.5 \mathrm{ml} / 5$ horas y egresos totales de 982.5 $\mathrm{ml} / 5$ horas con un balance total $+240 \mathrm{ml} / 5$ horas). Filtrado glomerular elevado (164); resultado esperado debido a la administración de diurético (Furosemide).

\section{Patrón Actividad-Ejercicio}

Apoyo ventilatorio ciclado por volumen y modo asisto controlado $\left(\mathrm{FiO}^{2}\right.$ de 50\%, Pi $16 \mathrm{~cm} \mathrm{H} 2 \mathrm{O}, \mathrm{Fr} 13 \mathrm{r} / \mathrm{min}$, I:E 1:2.5 y PEEP $8 \mathrm{cmH} 2 \mathrm{O}$ ). Eupneico (FR 13 por minuto) con adecuado desarrollo musculoesquelético y ángulos costales conservados, ausencia de retracción, abombamiento de los espacios intercostales y de sonidos adventicios; saturación de oxígeno adecuada (97\%). De acuerdo al índice de Kirby presenta lesión pulmonar aguda (270).

Tensión arterial $(100 / 60 \mathrm{mmHg})$ y frecuencia cardiaca dentro de parámetros normales (FC 100 por minuto) con una presión arterial media (PAM) dentro del rango normal $(73 \mathrm{mmHg})$ con un gasto cardíaco adecuado (4 litros). Ausencia de alteraciones en válvulas cardíacas; llenado capilar distal y proximal adecuado $(<3)$. Presencia de catéter venoso central en vena subclavia derecha con tres lúmenes, funcional con presencia de inflamación y rubor en zona circundante.

Mantiene posición en decúbito dorsal con una capacidad de movilización totalmente limitada; riesgo de caídas según escala de Morse en riesgo medio (50 puntos).

En cuanto a los exámenes de laboratorios clínicos de tiempo de coagulación, presenta tiempos de protrombina (T.P) alargados, relacionado con un problema en el hígado y tiene relación con lo expuesto en el patrón nutricional metabólico. En lo referente a la gasometría arterial presenta una alcalosis respiratoria (Tabla 3).

\section{Patrón Cognitivo-Perceptivo}

Inconsciente por acción farmacológica con puntuación máxima en escala de Ramsay (6 puntos); ausencia de respuesta a ruidos fuertes y en escala de RASS (-5 puntos) en puntuación mínima; sin respuesta a la voz o al estímulo físico. Ausencia de respuesta a reacción a la luz y a la acomodación; pupilas en estado de miosis. Presencia de sonda nasogástrica funcional y a derivación. Ausencia de dolor según escala de Campbell (0 puntos).
Tabla 1. Resultados de exámenes de laboratorio referentes al Patrón Nutricional Metabólico

\begin{tabular}{|c|c|c|}
\hline $\begin{array}{c}\text { Biometría Hemá- } \\
\text { tica }\end{array}$ & Resultados & $\begin{array}{c}\text { Valores de Re- } \\
\text { ferencia }\end{array}$ \\
\hline Hemoglobina & $12.5 \mathrm{gr} / \mathrm{dl}$ & $11.5-18$ \\
\hline Hematocrito & 39.3 & $35-52$ \\
\hline $\mathrm{HCM}$ & $26.2 \mathrm{pg}$ & $27-32$ \\
\hline Leucocitos & $\begin{array}{c}5.26 \\
\times 103 \mathrm{~mm} 3\end{array}$ & $4-10$ \\
\hline Neutrófilos & $85.5 \%$ & $45-70$ \\
\hline Linfocitos & $8 \%$ & $15-45$ \\
\hline Eosinófilos & $0.01 \times 103 \mathrm{~mm} 3$ & $0.05-0.5$ \\
\hline Basófilos & $0.02 \times 103 \mathrm{~mm} 3$ & $0.02-0.1$ \\
\hline Bioquímicos & Resultados & $\begin{array}{c}\text { Valores de Re- } \\
\text { ferencia }\end{array}$ \\
\hline Glucosa & $227 \mathrm{mg} / \mathrm{dl}$ & 74-109 \\
\hline Perfil hepático & Resultados & $\begin{array}{c}\text { Valores de Re- } \\
\text { ferencia }\end{array}$ \\
\hline Bilirrubina directa & $0.86 \mathrm{mg} / \mathrm{dl}$ & $0-0.25$ \\
\hline $\begin{array}{l}\text { Bilirrubina indi- } \\
\text { recta }\end{array}$ & $0.35 \mathrm{mg} / \mathrm{dl}$ & $0-0.85$ \\
\hline Bilirrubina total & $1.21 \mathrm{mg} / \mathrm{dl}$ & $0-1.1$ \\
\hline Proteínas Totales & Resultados & $\begin{array}{c}\text { Valores de Re- } \\
\text { ferencia }\end{array}$ \\
\hline Proteínas PT & $3.85 \mathrm{gr} / \mathrm{dl}$ & $6.7-8.7$ \\
\hline Albumina & $2.29 \mathrm{gr} / \mathrm{dl}$ & $3.8-5.1$ \\
\hline Serología & Resultados & $\begin{array}{c}\text { Valores de Re- } \\
\text { ferencia }\end{array}$ \\
\hline Procalcitonina & 16.08 & - \\
\hline
\end{tabular}

Tabla 2. Resultados de exámenes de laboratorio referentes al Patrón Eliminación

\begin{tabular}{|c|c|c|}
\hline $\begin{array}{l}\text { Química San- } \\
\text { guínea }\end{array}$ & Resultados & $\begin{array}{c}\text { Valores de Refe- } \\
\text { rencia }\end{array}$ \\
\hline Urea & $33 \mathrm{mg} / \mathrm{dl}$ & $16.6-48-5$ \\
\hline Creatinina & $0.79 \mathrm{mg} / \mathrm{dl}$ & $0.7-1.2$ \\
\hline $\begin{array}{l}\text { Electrolitos } \\
\text { Séricos }\end{array}$ & Resultados & $\begin{array}{c}\text { Valores de Refe- } \\
\text { rencia }\end{array}$ \\
\hline Sodio & $140 \mathrm{mmol} / \mathrm{L}$ & $135-145$ \\
\hline Potasio & $3.4 \mathrm{mmol} / \mathrm{L}$ & $3.6-5$ \\
\hline
\end{tabular}


Tabla 3. Resultados de exámenes de laboratorio referentes al Patrón Actividad y Ejercicio

\begin{tabular}{c|c|c}
\hline Biometría Hemática & Resultados & Valores de Referencia \\
\hline Plaquetas & $259 \times 103 \mathrm{~mm} 3$ & $150-480$ \\
\hline Tiempos de Coagulación & Resultados & Valores de Referencia \\
\hline T.P & $20 \mathrm{seg}$ & $11.8-15.5$ \\
T.T.P.A & $31.5 \mathrm{seg}$ & $20-40$ \\
\hline Gasometría Arterial & Resultados & Valores de Referencia \\
\hline $\mathrm{FiO}^{2}$ & $45 \%$ & - \\
$\mathrm{PO}^{2}$ & $135 \mathrm{mmHg}$ & $80-100 \mathrm{mmHg}$ \\
$\mathrm{HCO}_{3}$ & $20.4 \mathrm{mmol} / \mathrm{L}$ & $22-26$ \\
$\mathrm{pH}^{\mathrm{PaCO}}$ & 7.52 & $7.35-7.45$ \\
\hline
\end{tabular}

\section{Diagnósticos de enfermería (4).}

\section{Diagnósticos reales}

00030- Deterioro del intercambio de gases $\mathrm{r} / \mathrm{c}$ cambios en la membrana alveolo capilar (Distrés respiratorio agudo) r/c gasometría arterial anormal (Alcalosis respiratoria: $\mathrm{Ph}$ de $7.52, \mathrm{PaCO}^{2}$ de $135 \mathrm{mmHg}$ y $\mathrm{HCO}_{3}$ de $20.4 \mathrm{mmol} / \mathrm{L}$ ) y color anormal de la piel (Piel pálida).

00043- Protección ineficaz r/c perfiles hematológicos anormales (Neutrofilia, linfocitopenia y esosinopenia) m/p deficiencia inmunitaria (Procalcitonina elevada).

00044- Deterioro de la integridad tisular r/c factores mecánicos (Laparotomía exploratoria) m/p lesión tisular (Herida quirúrgica en fase inflamatoria).

00108- Déficit de autocuidado: baño r/c disminución del estado mental (Sedoanalgesia, Ramsay 6 y RASS - 5) m/p incapacidad para lavarse el cuerpo.

\section{Diagnósticos de riesgo}

00202- Riesgo de perfusión gastrointestinal ineficaz c/d diabetes mellitus (Tipo II), inestabilidad hemodinámica (Hipertensión intraabdominal grado II y disminución de perfusión abdominal) y efectos secundarios al tratamiento (Cirugía abdominal: laparotomía exploratoria).

00039- Riesgo de aspiración c/d depresión del reflejo tusígeno, depresión del reflujo nauseoso, intubación endotraqueal y disminución del nivel de conciencia (Sedoanalgesia, Ramsay seis y RASS - 5).

00206- Riesgo de sangrado c/d perfiles hematológicos anormales (T.P alargados).

00179- Riesgo de nivel de glucemia inestable c/d estado de salud física (DM II).

00178- Riesgo de deterioro de la función hepática c/d perfiles hematológicos anormales (Hiperbilirrubinemia).

00155- Riesgo de caídas c/d disminución del estado mental (Sedoanalgesia, Ramsay seis y RASS menos cinco). 


\section{PLAN DE CUIDADO DE ENFERMERÍA}

Dominio: Actividad/Reposo (4)

Clase: Respuestas cardiovasculares/pulmonares (4)

Diagnóstico enfermero (NANDA): 00029- Disminución del gasto cardíaco r/c alteración de la frecuencia cardiaca y alteración de la precarga m/p taquicardia (FC 100 por minuto), edema (Ambos pies fóvea una cruz) y disminución de la presión venosa central.

Objetivo: El usuario mejorará el gasto cardíaco posterior a las intervenciones de Enfermería durante su estancia hospitalaria en el servicio de UCl.

\begin{tabular}{l} 
Dominio: Salud fisiológica (II) Clase: Cardiopulomar ( E ) \\
\hline Resultado esperado (NOC): 0400- Efectividad de la bomba cardíaca \\
Definición: Adecuación del volumen de sangre expulsado del ventrículo izquierdo para apoyar la presión de per- \\
fusión sistémica.
\end{tabular}

\begin{tabular}{|c|c|c|c|c|c|c|c|}
\hline \multirow[t]{2}{*}{ Indicadores } & \multicolumn{2}{|c|}{ Puntuación Diana } & \multicolumn{5}{|c|}{ Escala (s) de medición } \\
\hline & $\begin{array}{l}\text { Mante- } \\
\text { ner a: }\end{array}$ & $\begin{array}{c}\text { Aumentar } \\
\text { a: }\end{array}$ & 1 & 2 & 3 & 4 & 5 \\
\hline 040001- Presión sanguínea sistólica & 3 & 4 & & & & & \\
\hline 041019- Presión sanguínea diastólica & 3 & 4 & Grave & & Mode- & Leve & Ningu- \\
\hline 040002- Frecuencia cardíaca & 3 & 4 & & cial & rada & & na \\
\hline 040003- Índice cardíaco & 5 & 5 & & & & & \\
\hline 040006- Pulsos periféricos & 5 & 5 & & & & & \\
\hline 040025- Presión venosa central & 1 & 4 & & & & & \\
\hline 040013- Edema periférico & 4 & 5 & & & & & \\
\hline 040029- Deterioro cognitivo & 1 & 1 & & & & & \\
\hline 040031- Palidez & 3 & 5 & & & & & \\
\hline
\end{tabular}

\section{Intervenciones (NIC) / Actividades}

6680- Monitorización de signos vitales:

- Monitorizar la presión arterial, pulso, temperatura y es tado respiratorio.

- Monitorizar ritmo y frecuencia cardíaca.

- Monitorizar la presencia y la calidad de los pulsos.

3480- Monitorización de las extremidades inferiores:

- Examinar la presencia de edema en las extremidades inferiores.

- Examinar el color, temperatura, la hidratación, la textura y grietas en la piel.

- Palpar pulso pedio y tibial posterior.

- Determinar el relleno capilar.

4150- Regulación hemodinámica:

- Realizar una valoración exhaustiva del estado hemodinámico: presión venosa central.

- Determinar el estado de perfusión.

- Administrar medicamentos inotrópicos.

- Administrar fármacos vasoconstrictores.

4120- Manejo de líquidos:

- Realizar un registro preciso de entradas y salidas.

- Vigilar el estado de hidratación.

- Administrar diuréticos.

\section{Fundamentación científica}

La función de la bomba cardíaca depende de la precarga, la poscarga, el estado contráctil que representa las características del desempeño del músculo independiente de las distintas condiciones de cargas y la frecuencia cardíaca. En la determinación del volumen sistólico intervienen la precarga, poscarga y la contractilidad; el volumen sistólico es directamente proporcional a la precarga e inversamente proporcional a la poscarga (8).

\section{Evaluación}

Durante los días de valoración presenta cambios en la tensión arterial con tendencia a la hipertensión (DIANA 3) y taquicardia (DIANA 3). En cuanto al índice cardíaco se encuentra una disminución al día 1/09/16, con un total de 1,800 litros, aumentando al día siguiente con 4 litros (DIANA 5). Los pulsos periféricos se mantienen perceptibles durante los días de la valoración y sin cambio alguno (DIANA 5), la palidez continua pero con una mejora al 2/09/16 (DIANA 4).

En cuanto a la presión venosa central se encuentra un aumento al día 1/09/16 con valores de $13 \mathrm{mmHgH}_{2} \mathrm{O}$, (DIANA 5) y el edema de ambos pies aumenta el día 1/09/16 a tres cruces y disminuye al día siguiente a dos (DIANA 3).

Referente a los medicamentos, se aumenta la dosis de Norepinefrina a de 1.5 a $2.0 \mathrm{ml} / \mathrm{hr}$ para el día 1/09/16 y disminuyen a la dosis principal al siguiente día, así mismo se comienza la administración de Amiodarona el 1/09/16 a $2.5 \mathrm{ml} / \mathrm{hr}$. El día 1/09/16 se comienza la infusión de Furosemide a $80 \mathrm{ml} / \mathrm{hr}$, aumentando a $100 \mathrm{ml} / \mathrm{hr}$ el mismo día y al día siguiente disminuyendo a $4 \mathrm{ml} / \mathrm{hr}$. 
Dominio: Eliminación e intercambio (3)

Clase: Función respiratoria (4)

Diagnóstico enfermero (NANDA): 00030- Deterioro del intercambio de gases $\mathrm{r} / \mathrm{c}$ cambios en la membrana alveolo capilar (Distrés respiratorio agudo) r/c gasometría arterial anormal (Alcalosis respiratoria: pH de 7.52, PaCO ${ }^{2}$ de 25 $\mathrm{mmHg}$ y HCO$_{3}$ de $20.4 \mathrm{mmol} / \mathrm{L}$ ), color anormal de la piel (Piel pálida) y taquicardia (FC 100 por minuto).

Objetivo: Mantendrá un adecuado estado respiratorio, posterior a las intervenciones del personal del servicio de UCl, durante su estancia en el servicio.

Dominio: Salud fisiológica (II) Clase: Cardiopulmonar ( E )

Resultado esperado (NOC): 0402- Estado respiratorio: intercambio gaseoso

Definición: Intercambio alveolar de $\mathrm{CO}^{2}$ y $\mathrm{O}^{2}$ para mantener las concentraciones de gases arteriales.

\begin{tabular}{|c|c|c|c|c|c|c|c|}
\hline \multirow[t]{2}{*}{ Indicadores } & \multicolumn{2}{|c|}{ Puntuación Diana } & \multicolumn{5}{|c|}{ Escala (s) de medición } \\
\hline & $\begin{array}{l}\text { Mante- } \\
\text { ner a: }\end{array}$ & $\begin{array}{c}\text { Aumen- } \\
\text { tar a: }\end{array}$ & 1 & 2 & 3 & 4 & 5 \\
\hline $\begin{array}{l}\text { 040208- Presión parcial } \\
\text { del oxígeno en la san- } \\
\text { gre arterial }\end{array}$ & 1 & 3 & Grave & Sustancial & Moderada & Leve & Ninguno \\
\hline $\begin{array}{l}\text { 040209- Presión parcial } \\
\text { de dióxido de carbono } \\
\text { en la sangre arterial }\end{array}$ & 1 & 3 & & & & & \\
\hline 040210- pH arterial & 5 & 5 & & & & & \\
\hline $\begin{array}{l}\text { 040211- Saturación de } \\
\text { O2 }\end{array}$ & 5 & 5 & & & & & \\
\hline
\end{tabular}

Intervenciones (NIC) / Actividades

7690- Interpretación de datos de laboratorios:

- Utilizar los rangos de referencia del laboratorio que esté realizando los análisis y analizarlos.

\section{0- Monitorización respiratoria:}

- Vigilar la frecuencia, ritmo, profundidad y esfuerzo de las respiraciones.

- Evaluar movimientos torácicos.

- Monitorizar patrones de respiración.

- Monitorizar niveles de saturación de oxígeno.

- Auscultar los sonidos respiratorios.

\section{0- Manejo de la ventilación mecánica invasiva:}

- Asegurarse de que las alarmas del ventilador estén conectadas.

- Comprobar regularmente las conexiones del ventilador.

- Administrar sedantes y analgésicos.

- Vigilar las lecturas del ventilador

1912- Manejo del equilibrio ácido básico alcalosis respiratoria:

- Obtener muestras para la gasometría arterial.

- Monitorizar la gasometría arterial.
Fundamentación científica

El aparato respiratorio comprende los pulmones, el sistema nervioso central, la pared torácica y la circulación pulmonar. El sistema nervioso central controla la actividad de los músculos de la pared costal que sirven de bomba para la respiración. Como estos componentes del aparato respiratorio actúan de forma concertada para lograr el intercambio gaseoso, el mal funcionamiento de uno de ellos o de su interrelación puede dar lugar a alteraciones de la función ${ }^{(9)}$.

\section{Evaluación}

Al día 31/09/16 según los nuevos laboratorios de gasometría arterial continúa presentando alcalosis respiratoria (PaC02 DIANA 1; pH arterial DIANA 5) con disnea y disociación torácico abdominal. De acuerdo al índice de Kirby, presenta un aumento a 300 al día 31/09/16, clasificándola en injuria pulmonar aguda y al día siguiente disminuye a 77 , en distrés respiratorio agudo; la saturación de oxígeno se encuentra dentro del rango normal durante todos los días (DIANA 5).

En cuanto a los parámetros del ventilador, son cambios al día 1/09/16 con un $\mathrm{FiO}^{2}$ de $45 \%$, Pi 15 cm H2 0 , Fr 8 1/min, los demás parámetros continúan igual. Al día siguiente se intenta cambiar a modo de Ventilación Intermitente Mandatorio Sincronizada con parámetros de $\mathrm{FiO}^{2}$ 45\%, Pi $16 \mathrm{~cm} \mathrm{H} 20$, Ti $1.33 \mathrm{seg}$, fTOT $14 \mathrm{~L}$, PEEP $8 \mathrm{~cm} \mathrm{H}_{2} \mathrm{O}, 1: \mathrm{E}$ de $1: 3$, P soporte de $12 \mathrm{~cm} \mathrm{H}_{2} \mathrm{O}$ y sensibilidad de $3 \mathrm{l} / \mathrm{min}$, sin embargo se presenta una respuesta disfuncional y se cambian nuevamente al modo $\mathrm{A} / \mathrm{C}$ con los siguientes parámetros: $\mathrm{FiO}^{2}$ 45\%, Pi $16 \mathrm{~cm} \mathrm{H}_{2} \mathrm{O}, \mathrm{Fr} 15$ 1/min, I:E 1:2.5, PEEP $8 \mathrm{~cm} \mathrm{H} 2 \mathrm{O}$. 
Dominio: Actividad/Reposo (4)

Clase: Respuestas cardiovasculares/pulmonares (4)

Diagnóstico enfermero (NANDA): 00202- Riesgo de perfusión gastrointestinal ineficaz c/d diabetes mellitus (Tipo II), inestabilidad hemodinámica (Hipotensión arterial, taquicardia, alteración de PVC, hipertensión intraabdominal grado II y disminución de perfusión abdominal) y efectos secundarios al tratamiento (Cirugía abdominal: laparotomía exploratoria).

Objetivo: Mantendrá una adecuada perfusión de órganos abdominales, posterior a los cuidados del personal de salud del servicio de $\mathrm{UCl}$, durante su estancia hospitalaria.

Dominio: Salud fisiológica (II) Clase: Cardiopulmonar ( E )

Resultado esperado (NOC): 0404- Perfusión tisular: órganos abdominales

Definición: Adecuación del flujo sanguíneo a través de los pequeños vasos de las vísceras abdominales para mantener la función orgánica.

\begin{tabular}{lccccccc}
\hline \hline Indicadores & \multicolumn{3}{c}{ Puntuación Diana } & \multicolumn{3}{c}{ Escala (s) de medición } \\
\cline { 2 - 7 } & $\begin{array}{c}\text { Mante- } \\
\text { ner a: }\end{array}$ & $\begin{array}{c}\text { Aumen- } \\
\text { tar a: }\end{array}$ & $\mathbf{1}$ & $\mathbf{2}$ & $\mathbf{3}$ & $\mathbf{4}$ & $\mathbf{5}$ \\
\hline $\begin{array}{l}\text { 040405- Ruidos intesti- } \\
\text { nales }\end{array}$ & 1 & 3 & Grave & Sustancial & Moderada & Leve & Ninguna \\
$\begin{array}{l}040421- \\
\text { función hepática }\end{array}$ & 4 & 5 & & & & \\
$\begin{array}{l}\text { 040413- Distención ab- } \\
\text { dominal }\end{array}$ & 1 & 3 & & &
\end{tabular}

\begin{tabular}{l}
\hline Intervenciones (NIC) / Actividades \\
\hline 6680 - Monitorización de signos vitales: \\
- Monitorizar la presión arterial, pulso, temperatura y estado \\
respiratorio.
\end{tabular}

- Monitorizar la presencia y la calidad de los pulsos.

\section{0- Manejo de líquidos:}

- Realizar un registro preciso de entradas y salidas.

- Vigilar el estado de hidratación.

\section{4- Cuidados de la sonda gastrointestinal:}

- Observar la correcta colocación de la sonda.

- Auscultar periódicamente los ruidos abdominales. - Monitorizar la cantidad, color y consistencia del contenido
drenado.

- Proporcionar cuidados de la piel alrededor de la zona de inserción.

\section{0- Administración de medicación:}

- Seguir las cinco reglas de la correcta administración de medicamentos.

- Verificar la orden médica antes de administrar

- Tomar nota de las alergias del usuario.

- Preparar la medicación utilizando equipos y técnicas apropiadas.

- Documentar la administración de los medicamentos.

\section{Fundamentación científica}

Los vasos sanguíneos del aparato digestivo forman parte de un sistema más extenso, llamado circulación esplácnica. Este sistema está formado por el flujo sanguíneo del tubo digestivo propiamente dicho más el correspondiente al bazo, al páncreas y al hígado. El diseño del sistema es tal que toda la sangre que atraviesa el intestino, el bazo y el páncreas fluye inmediatamente después hacia el hígado a través de la vena porta. En el hígado, la sangre pasa por millones de sinusoides hepáticos diminutos, para luego abandonar el órgano a través de las venas hepáticas, que desembocan en la vena cava de la circulación general. Este flujo sanguíneo secundario a través del hígado permite que las células reticuloendoteliales que revisten los sinusoides hepáticos eliminen las bacterias y otras partículas que podrían penetrar en la circulación general a partir del tubo digestivo, evitando así el acceso directo de los microorganismos potencialmente peligrosos al resto del cuerpo (10).

\section{Evaluación}

Al día 1/09/16 se encuentra un abdomen simétrico pero continua distendido (DIANA 3), además de una temperatura elevada al tacto. En cuanto a los ruidos intestinales estos se encuentran disminuidos; el día 1/09/16 aumenta a 1 por minuto y el día siguiente no se auscultan (DIANA 1).

La presión intraabdminal al día 1/09/16 disminuye a grado I (15 mmHg) y al día 2/09/16 aumenta a grado III (23 $\mathrm{mmHg}$ ). En cuanto a la perfusión abdominal se encuentra dentro del rango normal al día 1/09/16 con $61 \mathrm{mmHg}$ y al día siguiente continúa manteniéndose dentro de lo normal $(73 \mathrm{mmHg})$. 


\section{CONCLUSIONES}

El usuario hospitalizado en el servicio de $\mathrm{UCl}$ se encuentra sumamente vulnerable durante su estadía, por lo cual, es vital que el personal de enfermería implemente todos sus conocimientos y habilidades en la aplicación de sus intervenciones durante cada una de las etapas del $P E$, de esa manera se logrará brindar un cuidado enfermero de calidad y completamente fundamentado.

Es relevante recalcar que a pesar de la comunicación limitada con este tipo de usuario y del ambiente abrumador en el que uno se encuentra inmerso, es importante dedicar un tiempo específico para la persona, lo cual nos ayudará a conocerla y establecer un vínculo enfermera-usuario. Lo anterior es de suma importancia debido a que se podrá ofrecer seguridad y calidez, características que describen a nuestra profesión.

\section{REFERENCIAS BIBLIOGRÁFICAS}

1.Alfaro-LeFevre R. Aplicación del proceso enfermero: guía paso a paso. 4a ed. España: Springer-Verlag Ibérica; c1999. 274 p.

2.Madera Payeta A, Monasterio Martín I, Jaraiz Lara A, Cantador Gutiérrez R, Sánchez Sánchez J, Varas Moreno R. Estudio de casos [Internet]. España: UAM; [citado 29 septiembre 2017]. Disponible en https://www.uam.es/ personal_pdi/stmaria/jmurillo/InvestigacionEE/Presentaciones/Curso_10/EstCasos_Trabajo.pdf

3.Norma Oficial Mexicana NOM-025-SSA3-2013, Para la organización y funcionamiento de las unidades de cuidados intensivos. Diario Oficial de la Federación, (23-02-2012).

4.Herdman TH. NANDA Internacional. Diagnósticos Enfermeros: definición y clasificación. 2015-2017. Barcelona: Elsevier; c2015. 483 p.

5.Moorhead S, Johnson M, Maas M, Swanson E. Clasificación de resultados de enfermería (NOC). 5 a ed. España: Elsevier; c2014. 760 p.

6.Bulechek G, Bulecher H, Dochterman J, Wagner C. Clasificación de intervenciones de enfermería (NIC). 6 a ed. España: Elsevier; c2014. 664 p.

7.Norma Oficial Mexicana NOM-043-SSA2-2012, Servicios básicos de salud. Promoción y educación para la salud en materia alimentaria. Criterios para brindar orientación. Diario Oficial de la Federación, (22-012013).

8.Loscalzo J. Harrison: Neumología y cuidados intensivos. 1a ed. México: McGraw-Hill; c2013. 585 p.

9.Guyton C, Hall J. Guyton y Hall: Tratado de fisiología médica. 12a ed. España: Elsevier; c2006. 1174 p. 